\title{
A magnetic distance sensor with high precision
}

\author{
A. Zikmund, P. Ripka, \\ Czech Technical University in Prague, Faculty of Electrical Engineering, \\ Department of Measurement, Czech Republic, Prague
}

\begin{abstract}
A magnetic distance sensor working at very low frequency has been developed to measure distances up to $1 \mathrm{~m}$. The principle is similar to that of the 3-D magnetic tracker, but the system is optimized to minimize the error in distance for static measurements. The system is not affected by conductive objects. The uncertainty caused by noise and interference is below $2 \mathrm{~mm}$, even in a noisy environment. The measurement time of 3 minutes can be decreased to 1 minute, depending on the amplitude of the interferences and the required accuracy. After applying corrections, systematic error of $5 \mathrm{~mm}$ was achieved by using a calibration model. The system is scalable up to $20 \mathrm{~m}$ range.
\end{abstract}

\section{Introduction}

In [1], we described an implantable magnetic distance measurement system for stomach volume estimation. The system is based on one $2 \mathrm{~mm}$ diameter transmission coil and one detection coil, and it w orked at $3 \mathrm{kHz}$ frequency. The transmission coil excites the AC magnetic field, and the amplitude of the voltage induced in the detection coil depends on the distance between the coils. Its basic accuracy was $1 \mathrm{~mm}$ at $5 \mathrm{~cm}$ distance and $5 \mathrm{~mm}$ at $10 \mathrm{~cm}$ distance. The main source of error was angular mismatch and lateral displacement between the coils. These effects cannot be corrected in a singlesource, single-sensor system, and they can cause gross errors: in extreme cases (when the angular mismatch is $90^{\circ}$ ), the signal is completely lost.

In order to reduce this error below $10 \%$ for any angular position, we employed a 3-axial detection coil [2]. This approach greatly reduces the angular misalignment error, because the entire vector is known.

A similar system using AMR sensors is described in [3]. This system has additionally the source triplet and also a set of accelerometers to measure the roll and pitch angles of both the excitation coils and the sensor head. This forms a complete magnetic tracker, which allows all 6 unknown relative position parameters between the coils and sensors to be estimated ( 3 angular rotations and 3 spatial coordinates) by an AC generating magnetic field in a tri-axial coil source and by measuring this magnetic field with a tri-axial magnetometer. The range and accuracy of this system is limited mainly by the noise of the AMR sensor and also by other factors, such as geometrical errors of the transmission system and the sensor system. These systems use AC excitation usually at $\mathrm{kHz}$ frequencies, where the $1 / \mathrm{f}$ noise of magnetic sensors has a low value. Serious problems arise when a conducting object appears close to the system: eddy currents generated in the object cause distortion of the transmitted magnetic field, resulting in an error in position estimation. The amplitude of the eddy currents and the resulting error increase with the excitation frequency. A low-frequency magnetic tracker resistant to the presence of conducting objects is described in [4]. The weak point of that design is its low resistance against field interference. The improved version fuses the signal from inertial navigation sensors to aid during fast motion [5], but the static accuracy is about 3 to $5 \%$. Our goal was to develop a magnetic measuring system optimized for distance measurement which would work up to $1 \mathrm{~m}$. No dynamic tracking ability was required, which allowed us to use more effective filtering to suppress the magnetic noise from the environment. The system is similar to a magnetic 
tracker, but we utilize more precise fluxgate magnetic sensors and we have no strict requirements for angular precision. Another difference from traditional magnetic trackers is that we use a very low frequency field to avoid the above-mentioned problems with conductive objects. The main application of the system is in precise measurements of distances when other methods, e.g. ultrasound and lasers, fail because their signal is dissipated by the environment.

In the building industry and in archeology, magnetic methods are most often used for wall thickness measurements. In the mining industry, the requirement is to measure the distance between two drills for explosives. These holes are drilled in parallel, but in reality they are always inclined; the exact amount of explosive is calculated for each case from the measured distances. RF methods often fail in this application due to the presence of conductive objects.

\section{The principle of the magnetic tracker}

The arbitrary position between two parts of the magnetic tracker is defined by six variables. Fig. 1 shows a sketch of the spatial situation. The arbitrary position is characterized by distance, azimuth and elevation, which are the spatial coordinates of two points, and the mutual rotation yaw, pitch and roll of the two coordinate triplets.

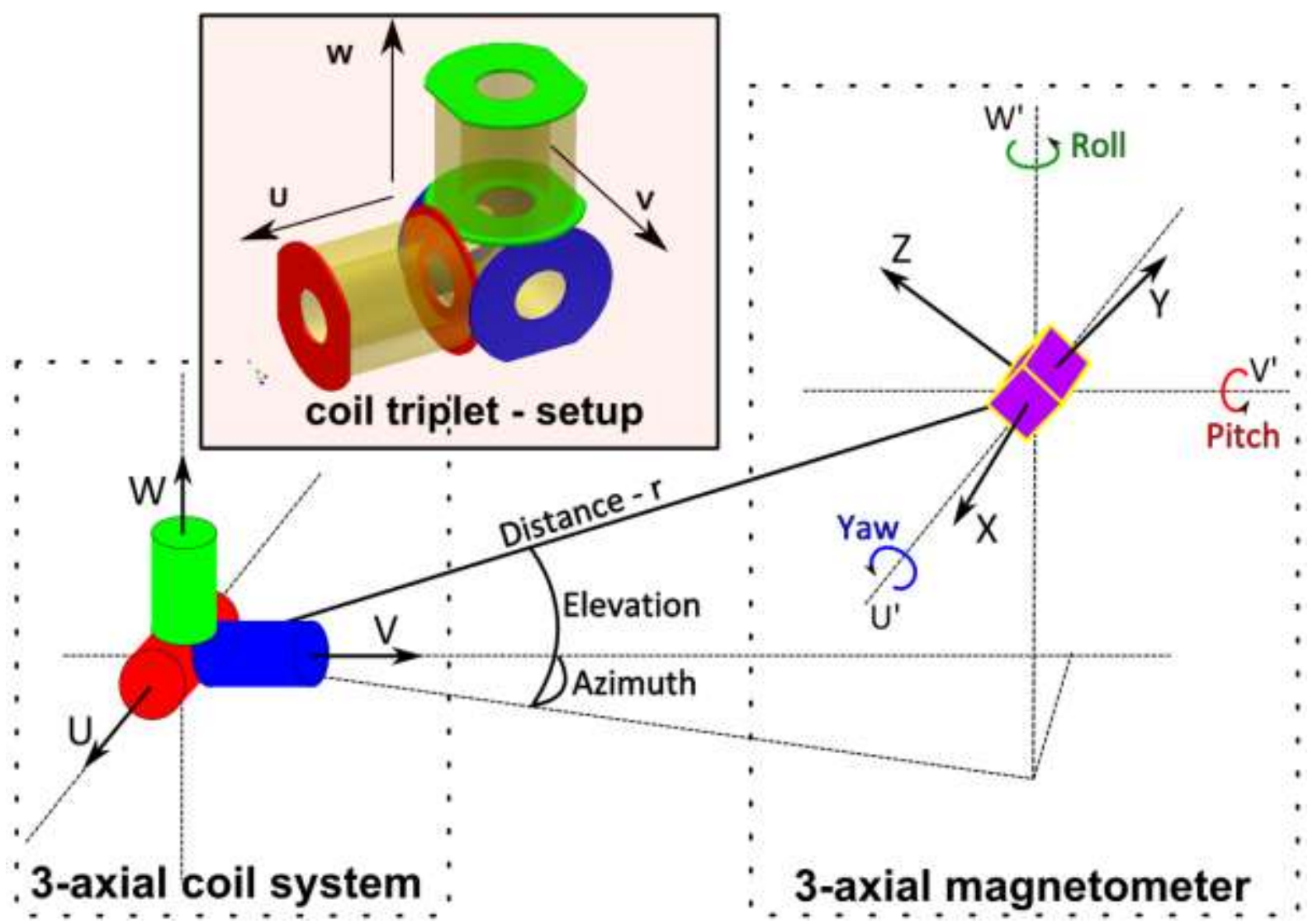

Fig. 1. Conceptual scheme of the distance meter and the real construction of transmission coil triplet $\mathrm{U}, \mathrm{V}, \mathrm{W}$.

Some simple magnetic trackers, e. g. the system described in [6], use a permanent magnet as a magnetic marker. An inherent problem is that the DC field of the marker cannot be distinguished from the Earth's field, which causes gross error in larger distances. 
We therefore use small solenoid coils as the excitation part of our magnetic tracker. The magnetic field of a small solenoid coil at distance $r$ larger than the coil size can be approximated by the formula for an ideal dipole with a magnetic moment of $m=N I A$,

where $N$ is the number of turns, $I$ is the excitation current, and $A$ is the average turn area. The value of $m$ should be measured, as $A$ cannot be estimated with the required precision. The radial component of the generated magnetic field is

$$
B_{r}=2 \mu_{0} m \cos \phi / 4 \pi r^{3}=\cos \phi \cdot 2 \cdot 4 \pi \cdot 10^{-7} \mathrm{NIA} / 4 \pi r^{3}=\cos \phi \cdot 2 \mathrm{NIA} \times 10^{-7} / r^{3}(T)=\cos \phi \cdot 200 \mathrm{NIA} / r^{3}(n T)
$$

and the tangential component is:

$$
B_{\phi}=\mu_{0} \operatorname{msin} \phi / 4 \pi r^{3}
$$

where $\phi$ is the angle between the coil axis and $r$ is the distance to the measured point.

Our source coils are $35 \mathrm{~mm}$ long, 22/35 mm diameter 300-turn solenoids mounted orthogonally. The coil shape is optimized to imitate the magnetic dipole [7]. With 1.5 A excitation current, each of them has a dipole moment of $0.287 \mathrm{Am}^{2}$. At a distance of $1 \mathrm{~m}$, the maximum theoretical field is $57 \mathrm{nT}$ in the axial direction (in the first Gauss position i.e. $\phi=0$ ). If we commutate the current polarity, the field step is double, i.e. $114 \mathrm{nT}$. At the same distance, the minimum field is $28 \mathrm{nT}$ (in the second Gauss position i.e. $\phi=\pi / 2$ ).

In our excitation coil system we use three orthogonal coils, so if one of them is in the second Gauss position another coil is in the first Gauss position. The resulting detection space of the tracker has the shape of a sphere with its radius given by the resolution of the magnetic sensor and by the level of noise reduction.

The second part of the magnetic tracker consists of a tri-axial magnetic sensor to obtain complete information on the direction of the generated magnetic field. To get a precise value of the measured distance the magnetic sensor should have low noise. Offset variations can be compensated by using bipolar excitation field. AMR sensors are small and cheap, but they have a noise level in the order of $5 \mathrm{nT}$ [8], which is too high for the required accuracy in long distance measurement. GMR sensors also do not achieve acceptable noise level. Even with AC excitation [10] their noise is in level of $15 \mathrm{nT}$ which is not sufficient for the precise distance meter.

The noise of GMI sensors is below 10pT RMS $/ \sqrt{ } \mathrm{Hz} @ 1 \mathrm{~Hz}$ [11] which would meet the requirements for the distance sensor precision, but their sensitivity and offset are strongly temperature dependent [12] and thus they are not suitable for this application.

We therefore used tri-axial fluxgate sensors (Billingsley TFM100G2) with resolution of $0.1 \mathrm{nT}$ and sufficient offset and gain stability. The noise power spectrum density is $\leq 12 \mathrm{pT}$ RMS $/ \sqrt{\mathrm{Hz}} @ 1 \mathrm{~Hz}$, which drops below $4 \mathrm{pT}$ at $10 \mathrm{~Hz}$. The offset drift of $\pm 0.6 \mathrm{nT} / \mathrm{K}$ is still large, but its effect can be suppressed by calculating the field differences. The sensitivity temperature dependence is only $0.007 \% / \mathrm{K}$, which makes the sensors ideal for this application. Another advantage of these sensors is their large linearity (linearity error below $\pm 0.015 \%$ of the Full Scale). A disadvantage of fluxgate sensors is their large size. Even though the sensors that we use are "miniature", the sensor head is approx. $3 \times 3 \times 3 \mathrm{~cm}$ in size, and there is a need for the corrections discussed in Section 3.2.

The resistance of the excitation coils is increased due to their heating. We used stabilized current sources to stabilize the current which defines the magnetic field that is created, and the currents were measured to calculate the real generated magnetic field created by the coil source. Heating also increased the coil size and thus the coil constant, due to thermal dilatation. In our case, the corresponding error was below $0.1 \%$, so that no corrections were necessary. However, this effect should be taken into consideration for other applications. 


\subsection{Excitation sequence}

During one excitation cycle, current square wave pulses of both polarities are sequentially sent to individual source coils U, V and W. In this way, we can easily remove the Earth's magnetic field vector and, by averaging from several cycles, we also can reduce the noise. To keep the eddy current effect low, the operating frequency is below $10 \mathrm{~Hz}$. The unknown distance and 5 angles can be calculated from the 9 measured field differences $\triangle \mathrm{BXU}, \triangle \mathrm{BXV}, \triangle \mathrm{BXW}, \ldots, \Delta \mathrm{BZW}$. In our case, we concentrated on evaluating distance $r$. We therefore optimized the estimation procedure to minimize the uncertainty of this parameter. In fact, we reduced the magnetic position tracker to the magnetic distance sensor. In this paper we do not discuss how accurately the position angles are evaluated.

\subsection{Calculation}

With respect to the non-linear equations describing the dipole magnetic field, the solver calculates the position from 9 values of the measured magnetic field. The algorithm is based on the LevenbergMarquardt non-linear optimization method [7]. This method converges from the initial point to the corresponding result. However, due to the symmetry of the magnetic field dipole we have to consider the non-unique solution of the numerical algorithm, which can converge to the opposite point from the real position. In our special case we find only the distance, which is not influenced by this problem.

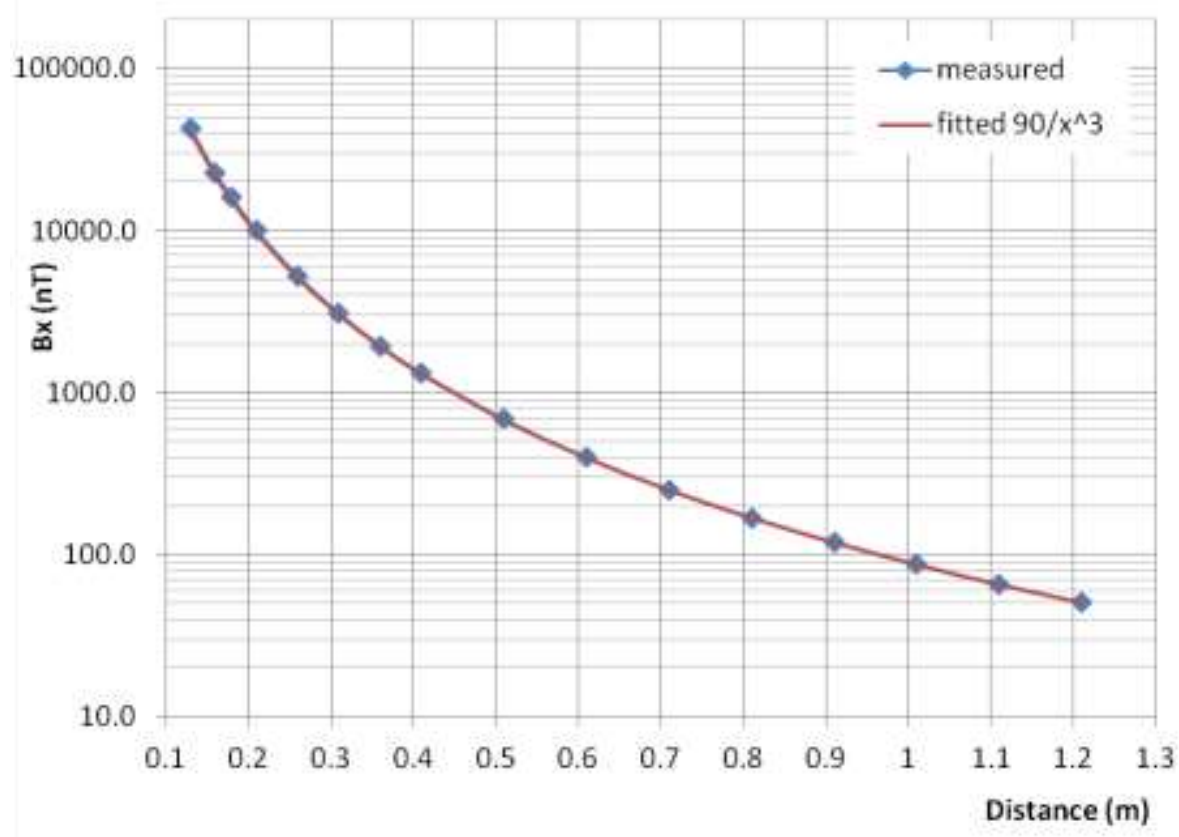




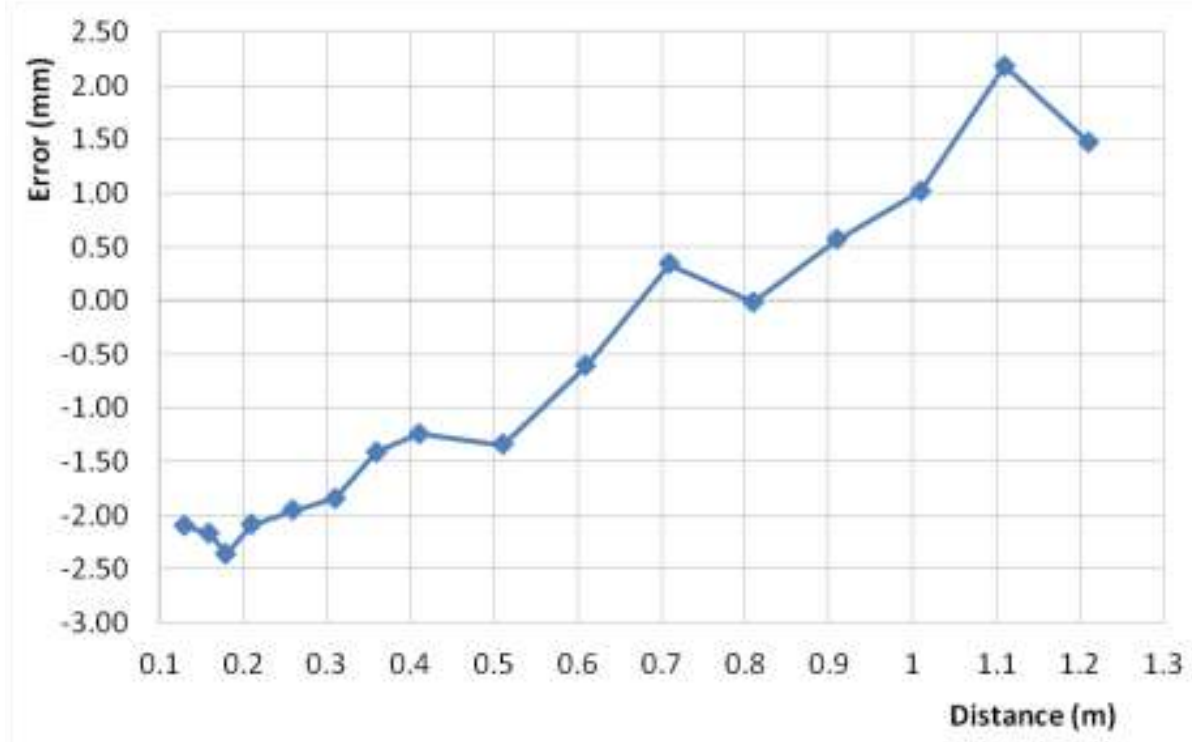

Fig. 2. (a) Axial field as a function of distance. The dipole moment of the source was 0.287Am2. (b) Error in distance estimation for the 1-axial case, measured in a building environment, measurement time 3 min.

\section{Measured values}

\subsection{Verification of the dipole approximation}

To verify the precision of the approximation of the solenoid by the magnetic dipole, a test was carried out in the first Gauss position. This measurement setup was based on a single coil, which was periodically excited by current with both polarities, and the magnetic sensor was precisely aligned with the magnetic axis of the coil (sensor aligned axially to the coil). The measured magnetic field with respect to the distance is shown in Fig. 2a. The measured data was fitted to $s / r^{3}$ dependence. The sensitivity constant $s$ (which depends on the actual constant of the transmission coil and the sensitivity of the sensor) was found by the least square method to minimize the total error in distance. This explains the fact that the resulting error is zero at mid-distance. The error is mainly caused by the nondipole character of the magnetic field in the close vicinity of the solenoid. To increase the accuracy of the device further, this error can be compensated by more precise approximation of the near field; however for our application this was not necessary. Fig. $2 b$ shows the resulting error. The absolute error was below $2 \mathrm{~mm}$. It should be noted that for larger distances the measured field steps were extensively averaged to reduce the noise.

The main noise sources are the external environment (mainly electric currents and movement of ferromagnetic objects) and the own noise of the sensor. In our case, the sensor noise was in the range of $10 \mathrm{pT} / \sqrt{\mathrm{Hz}} @ 1 \mathrm{~Hz}$, which was negligible compared to the environmental noise.

The influence of the sensor offsets and their temperature drifts, which is normally the most severe problem of magnetic sensors, is suppressed by commutation of the source coil currents.

The magnetic distance sensor will be used in magnetically noisy environments. For a typical 3minute data file containing 200 sets of six tri-axial measurements (Fig. 3), the RMS noise of the external field was 64, 98 and $389 \mathrm{nT}$ in the $\mathrm{X}, \mathrm{Y}$ and $\mathrm{Z}$ directions, respectively (calculated as the standard deviation of the 200 values of the measured magnetic field). This noise was reduced by calculating the differences between the subsequent readings $\mathrm{B}^{+}$and $\mathrm{B}^{-}$for the positive and negative pulse, and only then averaging these differences, e.g. 


$$
\Delta B_{x}=\frac{1}{k} \sum_{n=1}^{k}\left(B_{n}^{+}-B_{n}^{-}\right)
$$

As we flip the field polarity 10 times each second, slower field variations are suppressed. After this type of averaging, the resulting RMS error of our sample dataset is only 1.5, 4.3 and 12.8 nT. The uncertainty can be further reduced below $1 \mathrm{nT}$ by longer averaging. These are values typical for a city environment. The field variations in a rural area and in an industrial environment may vary by an order of magnitude.

\subsection{Measurement of 3-D arbitrary position}

If the distance is measured in 3-D arbitrary position, the situation is much more complicated. We carried out a test when the sensor head is rotated in the horizontal plane. The sensor was placed at a distance of $0.5 \mathrm{~m}$. The magnetic fields generated by the 3D coil system are shown in Fig. 4. Each point represents the magnetic field at a specific horizontal angle after averaging 200 difference values between positive and negative excitation. The non-orthogonality of both source coils and magnetic sensors can be calculated from similar data.

In ideal cases, the magnetic field as a function of the azimuth angle should be a sine wave, but this is clearly not the case. We have analyzed systematic errors which can affect the measured values, namely:

- differences between the coil dipole moments

- differences between the individual sensor sensitivities

- angular deviations of both triplets from orthogonality

- the excitation coils have non-zero size (see Fig. 1) and they are not concentric; the same applies for the sensors

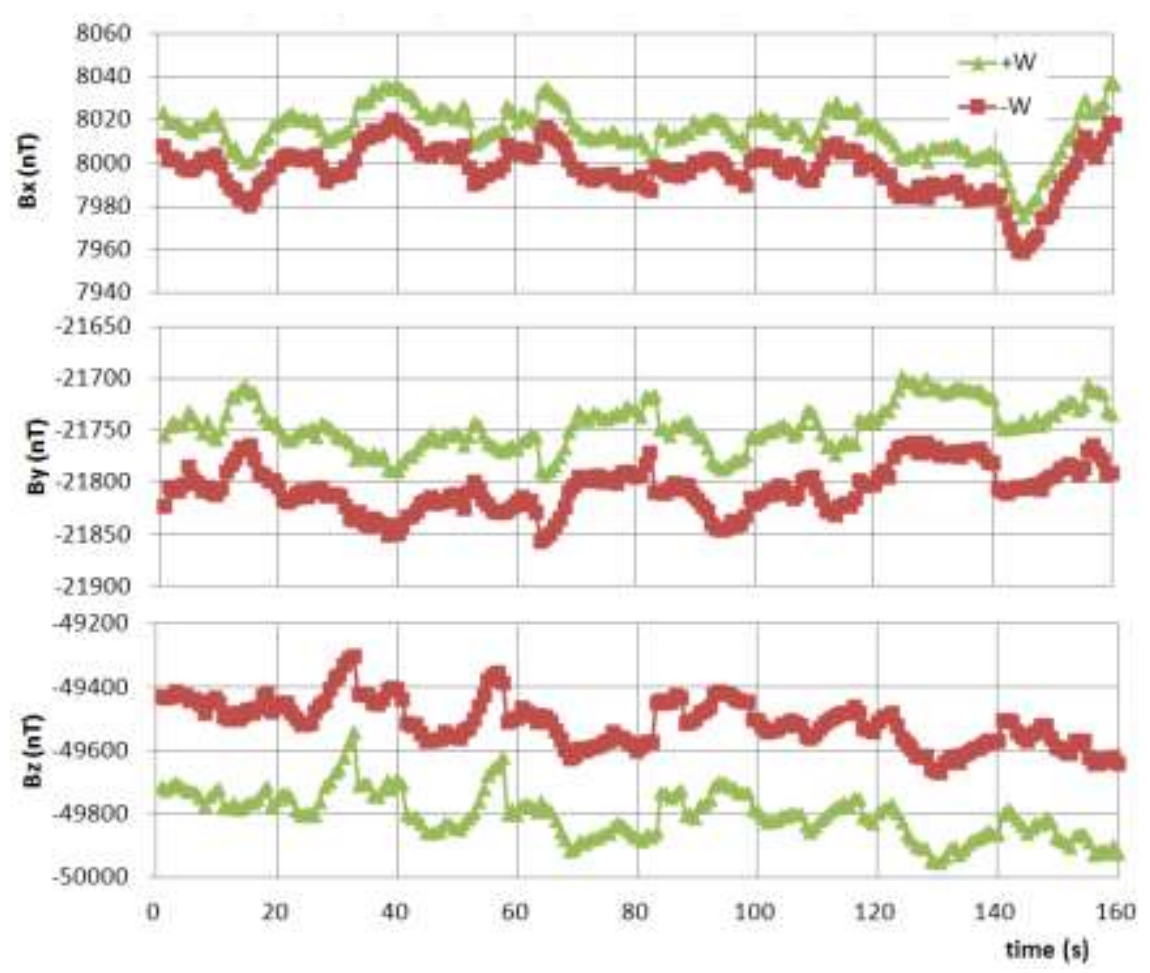

Fig. 3. The magnetic field measured at a distance of $0.5 \mathrm{~m}$ when I+ and I- currents are applied to the $\mathrm{W}$ coil 

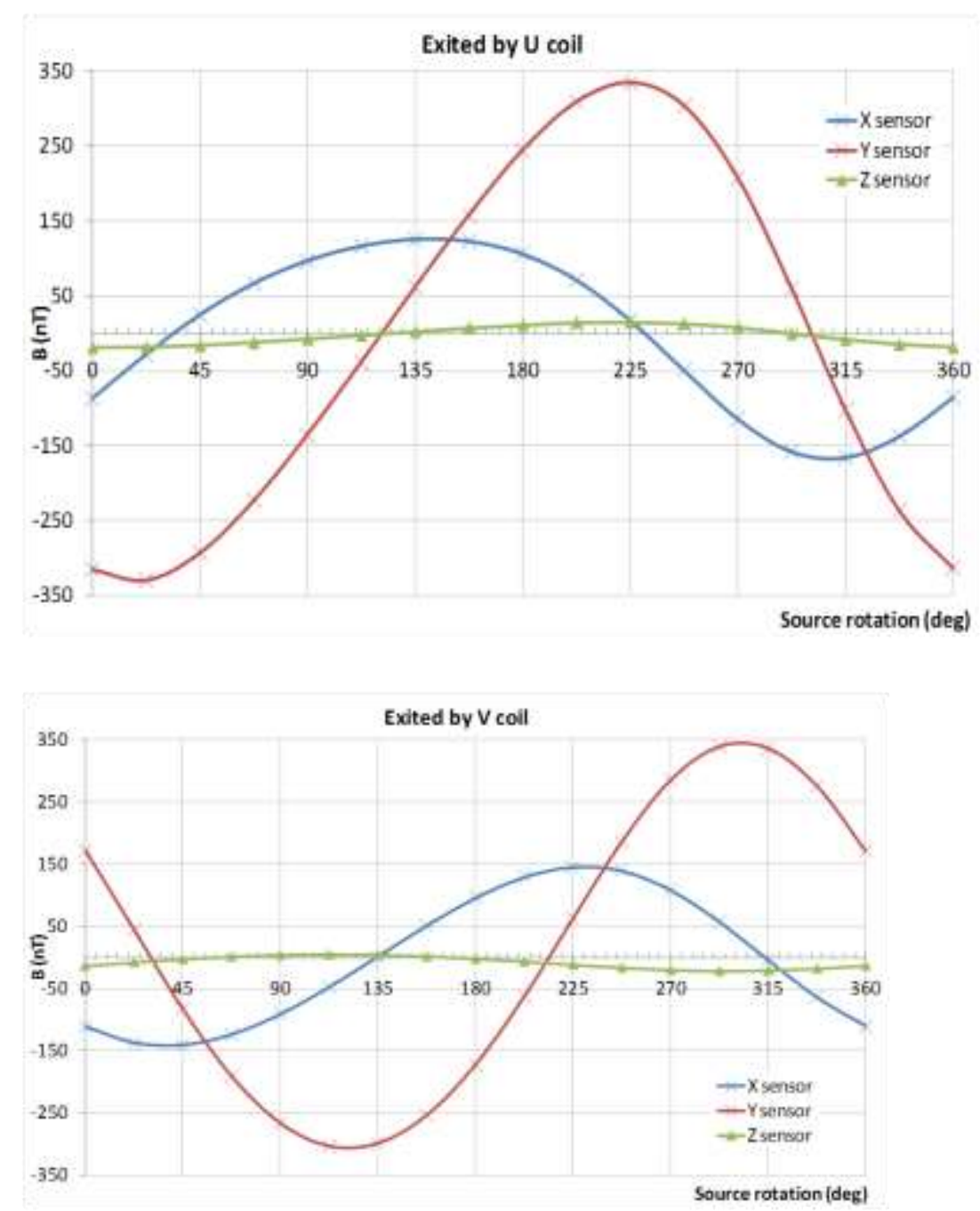

Fig. 4. (a) Signals from coil U rotated in the horizontal plane, sensor head at a dis tance of $50 \mathrm{~cm}$. (b) Signals from coil V rotated in the horizontal plane, sensor head at a distance of $50 \mathrm{~cm}$.

All these systematic errors can be corrected by calculation and subsequently by calibration.

In this paper, we have experimentally revealed only the main sources of error, and have compensated them. Fig. 5 shows an example of the calibration test: the sensor triplet was rotated in the horizontal plane, while the distance from the coil triplet was kept constant. First, the distance was calculated without corrections assuming that all coils are identical and their centers coincide in the same point. We observed a $25 \mathrm{~mm}$ variation in the calculated distance with rotational angle (blue curve). Then we added into the equation the correction element for the non-coincided centers of the coils and calculated the distance, which gave $15 \mathrm{~mm}$ variation (red curve). Finally, we obtain the distance with the corrected equations which incorporated also the fact that the coils have different magnetic moment.. The variation of the calculated distance was reduced to $9 \mathrm{~mm}$ (green curve). The clear harmonic components in the residual variation are caused by non-orthogonalities. After correction for all mentioned effects, the final error was decreased to $5 \mathrm{~mm}$ at a distance of $0.5 \mathrm{~m}$. The other imperfections contributed a maximum of $1 \%$ error, which is acceptable for many applications. 


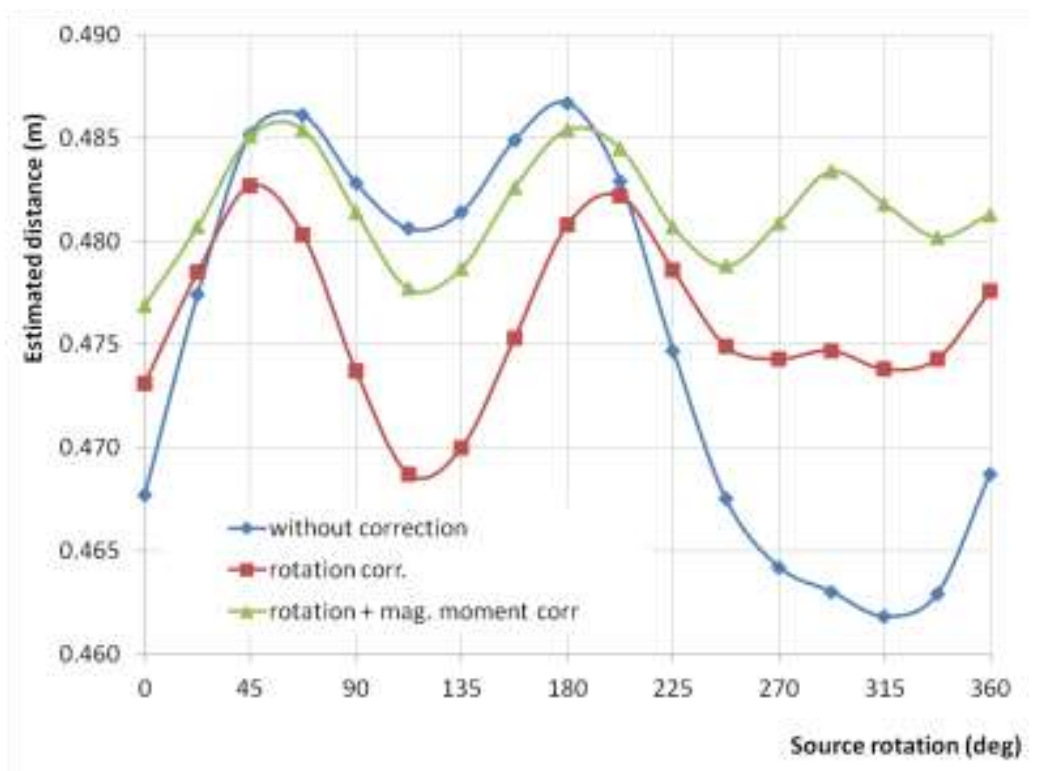

Fig. 5. The calculated distance, without applied correction and with applied correction.

\section{Conclusions}

Using a small tri-axial solenoid excitation (source) coil system powered by a 1.5 A current sequence, we achieved $1 \%$ precision in measuring distances up to $1 \mathrm{~m}$. Over larger distances or in a noisy environment such precision requires extensive averaging, and this may significantly increase the basic measurement time of 1 second. For much larger distances, the excitation coils should be scaled up and the current increased. The maximum realistic range for industrial applications is 20 to $50 \mathrm{~m}$. For this purpose, we estimate the magnetic moment of the coils to be about $10 \mathrm{Am}^{2}$, which gives us a sufficient magnetic field (about $10 \mathrm{nT}$ in 20 meters) for a 0.5 meter distance error. This means that a 1000-turn coil should have a cross sectional area $\mathrm{A}$ of $100 \mathrm{~cm}^{2}$, and an excitation current of $1 \mathrm{~A}$.

\section{References}

[1] J. Tomek J, P. Mlejnek, M. Janasek, et al.: Gastric motility and volume sensing by implanted magnetic sensors, Sensor Letters 5 (2007), 276-278

[2] J. Tomek, P. Mlejnek, V. Janásek, P. Ripka, P. Kašpar and J. Chen: The precision of gastric motility and volume sensing by implanted magnetic sensors, Sensors and Actuators A 142 (2008):3439.

[3] Liu Y., Y. Wang, D. Yan, Y. Zhou: DPSD Algorithm for AC Magnetic Tracking System, IEEE conf. Virtual Environments Human-Computer Interface and Meas. Syst., (2004): 101-106.

[4] H. M. Schepers, D. Roetenberg, P. H. Veltink: Ambulatory human motion tracking by fusion of inertial and magnetic sensing with adaptive actuation, Med Biol Eng Comput (2010) 48:27-37

[5] D. Roetenberg, P. Slycke, A. Ventevogel, P.H. Veltink: A portable magnetic position and orientation tracker, Sens. Actuat. A 135, (2007) 426-432[6] T. Liu, B. Wang: Study of magnetic ranging technology in horizontal directional drilling, Sensors and Actuators A (2011), in press, doi:10.1016/j.sna.2011.08.016

[7] E. Paperno, A. Plotkin: Cylindrical induction coil to accurately imitate the ideal magnetic dipole, Sens. Actuators A112 (2004) 248-252.

[8] P. Ripka, M.Vopalensky, A.Platil, M. Doscher, K.-M.H. Lenssen, H.Hauser: AMR magnetometer, Journal of Magnetism and Magnetic materials 254-255 (2003), 639-641 
[9] D. Marquardt: An Algorithm for Least-Squares Estimation of Nonlinear Parameters. SIAM J. Appl. Math. 11 (1963), 431-441.[10] P. Ripka, M. Todra, J. Stokes, R. Beech: AC-driven AMR and GMR magnetoresistor, Sensors and Actuators A: Physical (1999), 225-230

[11] L. Ding, S. Saez, C. Dolabdjian: Equivalent Magnetic Noise Limit of Low-Cost GMI Magnetometer, In IEEE SENSORS JOURNAL 9 (1-2) (2009), 159-168[12] M. Malátek, P. Ripka, L. Kraus: Temperature offset drift of GMI sensors, Sensors and Actuators A: Physical, Volume 147, (2008), 415-418

Fig 1. Conceptual scheme of the distance meter and the real construction of transmission coil triplet $\mathrm{U}, \mathrm{V}, \mathrm{W}$.

Fig 2. (a) Axial field as a function of distance. The dipole moment of the source was $0.287 \mathrm{Am}^{2}$

Fig 2. (b) Error in distance estimation for the 1-axial case, measured in a building environment, measurement time 3 minutes.

Fig 3. The magnetic field measured at a distance of $0.5 \mathrm{~m}$ when $+\mathrm{I}$ and $-\mathrm{I}$ currents are applied to the $\mathrm{W}$ coil.

Fig 4. (a) Signals from coil $U$ rotated in the horizontal plane, sensor head at a distance of $50 \mathrm{~cm}$.

Fig 4. (b) Signals from coil V rotated in the horizontal plane, sensor head at a distance of $50 \mathrm{~cm}$.

Fig 5. The calculated distance, without applied correction and with applied correction. 